Second Meeting, 8th December 1899.

R. F. Murrhead, Esq., M.A., B.Sc., President, in the Chair.

\title{
On the Evaluation of a certain Determinant.
}

By Lawrence Crawford, M.A., D.Sc.

1. In a paper by Mr Arthur Berry, M.A., in the Proceedings of the Cambridge Philosophical Society, Volume X. Pt. I., "On the Evaluation of a certain Determinant which occurs in the mathematical theory of statistics and in that of elliptic geometry of any number of dimensions," a remark is made that in the case of $n=3$ this determinant was readily evaluated by me by means of the formulæ of spherical trigonometry. I have thought that it might be of interest to show this evaluation, but $I$ shall merely state the determinant at once of order $\hat{3}$, and leave the reader to refer to the paper quoted for the general determinant.

2. The question then is, to evaluate the determinant

$$
\frac{\partial\left(\xi_{12}, \xi_{13}, \xi_{23}\right)}{\partial\left(r_{12}, r_{13}, r_{23}\right)}
$$

where $\xi_{12}=R_{12} / \sqrt{\mathbf{R}_{11} \mathbf{R}_{22}}$, \&c., where $R_{p q}$ is the minor (with proper sign) of the element in the $p$ th row and $q$ th column of $R$, where

$$
\mathbf{R}=\left|\begin{array}{lll}
1 & r_{12} & r_{13} \\
r_{12} & 1 & r_{23} \\
r_{13} & r_{23} & 1
\end{array}\right|
$$

3. We have $\mathbf{R}_{11}=1-r_{23}^{2}, \quad \mathbf{R}_{22}=1-r_{13}^{2}, \quad \mathrm{R}_{33}=1-r_{12}^{2}$;

$$
\mathrm{R}_{12}=r_{13} r_{23}-r_{12}, \quad \mathrm{R}_{13}=r_{12} r_{13}-r_{13}, \quad \mathrm{R}_{23}=r_{12} r_{13}-r_{23} \text {. }
$$

Put then $r_{23}=\cos a, r_{13}=\cos b, r_{12}=\cos c$, where $a, b, c$ are the sides of a spherical triangle, then $\mathbf{R}_{11}=\sin ^{2} a, \mathbf{R}_{22}=\sin ^{2} b, \mathbf{R}_{33}=\sin ^{2} c$, 
and $\mathbf{R}_{12}=\cos b \cos a-\cos c=-\sin a \sin b \cos C, \quad R_{13}=-\sin a \sin c \cos B$, and $R_{23}=-\sin b \sin c \cos A$,

$$
\therefore \xi_{12}=-\cos \mathrm{C}, \quad \xi_{13}=-\cos \mathrm{B}, \quad \xi_{23}=-\cos \mathrm{A} .
$$

$\therefore$ we wish $J$, where $J=-\frac{\partial(\cos C, \cos B, \cos A)}{\partial(\cos c, \cos b, \cos a)}$

$$
\begin{gathered}
=-\frac{\partial(\cos \mathrm{A}, \cos \mathrm{B}, \cos \mathrm{C})}{\partial(\cos a, \cos b, \cos c)} ; \\
\therefore \quad J \times \frac{\partial(\cos a, \cos \mathrm{b}, \cos c)}{\partial(a, b, c)}=-\frac{\partial(\cos \mathrm{A}, \cos \mathrm{B}, \cos \mathrm{C})}{\partial(a, b, c)} .
\end{gathered}
$$

In this $a, b, c$ are independent, $\mathbf{A}, \mathrm{B}, \mathbf{C}$ functions of $a, b, c$.

$$
\begin{aligned}
& \cos A=(\cos a-\cos b \cos c) / \sin b \sin c, \\
\therefore \quad \frac{\partial(\cos A)}{\partial a}= & -\sin a / \sin b \sin c
\end{aligned}
$$

and $\frac{\partial(\cos A)}{\partial b}=\left\{\sin ^{2} b \cos c-(\cos a-\cos b \cos c) \cos b\right\} / \sin ^{2} b \sin c$, $=(\cos c-\cos a \cos b) / \sin ^{2} b \sin c$, $=\sin a \cos C / \sin b \sin c$,

and $\frac{\partial(\cos A)}{\partial c}=\sin a \cos B / \sin b \sin c$.

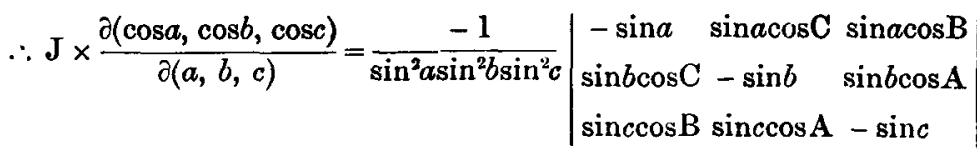

$$
=\frac{-1}{\sin a \sin b \sin c}\left|\begin{array}{ccc}
-1 & \cos \mathrm{C} & \cos \mathrm{B} \\
\cos \mathrm{C} & -1 & \cos \mathrm{A} \\
\cos \mathrm{B} & \cos \mathrm{A} & -1
\end{array}\right|
$$

$=-\left(-1+2 \cos \mathrm{A} \cos \mathrm{B} \cos \mathrm{C}+\cos ^{2} \mathrm{~A}+\cos ^{2} \mathrm{~B}+\cos ^{2} \mathrm{C}\right) / \sin a \sin b \sin c$ $=\left(1+2 \cos a^{\prime} \cos b^{\prime} \cos c^{\prime}-\cos ^{2} a^{\prime}-\cos ^{2} b^{\prime}-\cos ^{2} c^{\prime}\right) / \sin a \sin b \sin c$

(using the polar triangle)

$$
\begin{aligned}
& =\sin ^{2} \mathrm{~A}^{\prime} \sin ^{2} b^{\prime} \sin ^{2} c^{\prime} / \sin a \sin b \sin c \\
& =\sin ^{2} a \sin ^{2} \mathrm{~B} \sin ^{2} \mathrm{C} / \sin a \sin b \sin c \\
& =\sin ^{2} \mathrm{~A} \sin \mathrm{B} \sin \mathrm{C} / \sin a .
\end{aligned}
$$


Now $\frac{\partial(\cos a, \cos b, \cos c)}{\partial(a, b, c)}=\left|\begin{array}{ccc}-\sin a & 0 & 0 \\ 0 & -\sin b & 0 \\ 0 & 0 & -\sin c\end{array}\right|=-\sin a \sin b \sin c$

$\therefore \quad J=-\sin ^{2} \mathrm{~A} \sin \mathrm{B} \sin \mathrm{C} / \sin ^{2} a \sin b \sin c$

$=-(\sin \mathrm{A} / \sin a)^{4}$.

Also $\mathrm{R}=1-\cos ^{2} a-\cos ^{2} b-\cos ^{2} c+2 \cos a \cos b \cos c$

$=\sin ^{2} A \sin ^{2} b \sin ^{2} c$;

$\therefore \quad J=-\left(1-\cos ^{2} a-\cos ^{2} b-\cos ^{2} c+2 \cos a \cos b \cos c\right)^{2} / \sin ^{4} a \sin ^{4} b \sin ^{4} c$;

$\therefore \quad J=-R^{2} / R_{11}^{2} R_{22}^{2} R_{33}^{2}$. 